\title{
Genome-Wide Identification of Two-Component Signal Transduction System Genes in Melon (Cucumis melon L.)
}

\author{
Panjing Liu1,2,3,4, Xiaoyu Yang5, Yana Zhang1,2,3,4, Shuoshuo Wang1,2,3,4, Qian Ge ${ }^{1,2,3,4}$, Qiang Li, 1,2,3,

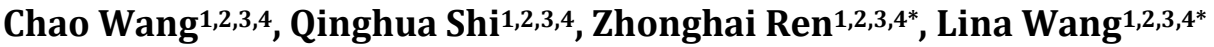 \\ ${ }^{1}$ State Key Laboratory of Crop Biology, Tai'an, China \\ ${ }^{2}$ Key Laboratory of Biology and Genetic Improvement of Horticultural Crops (Huanghuai Region), Tai'an, China \\ ${ }^{3}$ Shandong Collaborative Innovation Center for Fruit and Vegetable Production with High Quality and Efficiency, Tai'an, China \\ ${ }^{4}$ College of Horticulture Science and Engineering, Shandong Agricultural University, Tai'an, China \\ ${ }^{5}$ College of Life Sciences and Oceanography, Shenzhen University, Shenzhen, China \\ Email: *lnwangsdau@hotmail.com, ^zhren@sdau.edu.cn
}

How to cite this paper: Liu, P.J., Yang, X.Y., Zhang, Y.N., Wang, S.S., Ge, Q., Li, Q., Wang, C., Shi, Q.H., Ren, Z.H. and Wang, L.N. (2018) Genome-Wide Identification of Two-Component Signal Transduction System Genes in Melon (Cucumis melon L.). Agricultural Sciences, 9, 469-479.

https://doi.org/10.4236/as.2018.94032

Received: March 15, 2018

Accepted: April 27, 2018

Published: April 30, 2018

Copyright (c) 2018 by authors and Scientific Research Publishing Inc. This work is licensed under the Creative Commons Attribution International License (CC BY 4.0).

http://creativecommons.org/licenses/by/4.0/

\begin{abstract}
Two-component system (TCS) is responsible for cytokinin signaling, which plays critical roles in plant development and physiological process. This system is generally composed of two signaling factors, a histidine kinase (HK) and a response regulator (RR) that is associated with a histidine phosphotransfer (HP) protein. In this study, we performed systematic investigation on TCS genes in melon (Cucumis melon L.). We identified 44 TCS genes in melon, including $18 H K(L) s$ (9 HKs and 9 HKLs), 5 HPs (4 authentic and 1 pseudo), and 21 RRs (7 Type-A, 8 Type-B, and 6 pseudo). The classification and structure of these melon TCS members were introduced in detail as well. Our results provided new insights into the characteristics of the melon TCS genes and might benefit their functional study in future.
\end{abstract}

\section{Keywords}

Cytokinin, Melon, Two-Component System

\section{Introduction}

Cytokinins are essential for many of the physiological and developmental processes such as seed germination, functional root nodule establishment, lateral root development, shoot apical meristem maintenance, leaf expansion, flowering, circadian clock, nutrient mobilization, abiotic stress, and senescence [1] [2] [3] [4]. In eukaryotes such as yeast and plant, a two-component system (TCS) 
has been reported for the transduction of cytokinin signal [5] [6]. This TCS consists of two signaling factors, a histidine kinase $(H K)$ gene family and a response regulator $(R R)$ gene family [7]. HK can sense the cytokinin signals by phosphorylating its conserved histidine residues. The phosphoryl group is then transferred to a conserved asparagine residue on the receiver $(\mathrm{Rec})$ domain of an $\mathrm{RR}$, which modulates the activity of concerned downstream genes directly or indirectly [1] [7]. In addition, histidine phosphotransfer ( $H P$ ) genes are regarded as the mediators for the transfer of the phosphoryl group between the HKs and the RRs (Figure 1) [6] [8] [9].

Melon (Cucumis melon L.) is an economically important fruit crop that originates from Asian, with an average production during the past decade more than 29 million tons per year (FAOSTAT, 2017;

http://www.fao.org/faostat/en/\#home). This crop is mainly cultivated in tropical and temperate countries, especially in the Asian countries, with China leading the list [10] [11]. Although melon is a eudicot of interest for its specific biological properties [10] [12], there is still no genome-wide investigation on melon TCS genes. In this study, we examined the putative TCS genes and revealed that the melon genome contained a total of 44 members. Their classification and characteristics were also analyzed systematically. Our comprehensive analysis of the TCS genes might provide a framework for future functional dissection of TCSs in melon hormone signal transduction.

\section{Materials and Methods}

\subsection{Data Collection}

Protein sequence data of Arabidopsis AHKs, AHPs and ARRs were downloaded from the NCBI databases (https://www.ncbi.nlm.nih.gov/). Genome sequence data of melon deposited in the website of Cucurbit Genomics Data (http://cucurbitgenomics.org/organism/3) were used for TCS gene identification and analysis.

\subsection{Identification of the Putative Melon TCS Genes}

Previously cucumber and watermelon TCS members have been successfully identified by using Arabidopsis TCS genes [13]. So we also used Arabidopsis TCS protein sequences as queries to search for the putative counterparts in melon by BLASTP with E-value of 1e-5 [14]. The Pfam (http://pfam.janelia.org) and SMART (http://smart.embl-heidelberg.de/) tools were used to check whether these genes contained the structural characteristics and conserved domains of TCS elements, i.e., HisKA (Histidine Kinase A phosphoacceptor) domain, HATPase (histidine kinase-like ATPase) domain, Hpt (histidine-containing phosphotransfer) domain, and Rec domain. Information of abbreviation notation used in this article was listed in Table A1. Thereafter the identity of melon TCS genes with Arabidopsis was analyzed by BLASTP against Arabidopsis databases in TAIR (http://www.arabidopsis.org/). Their CDS and protein sequences 


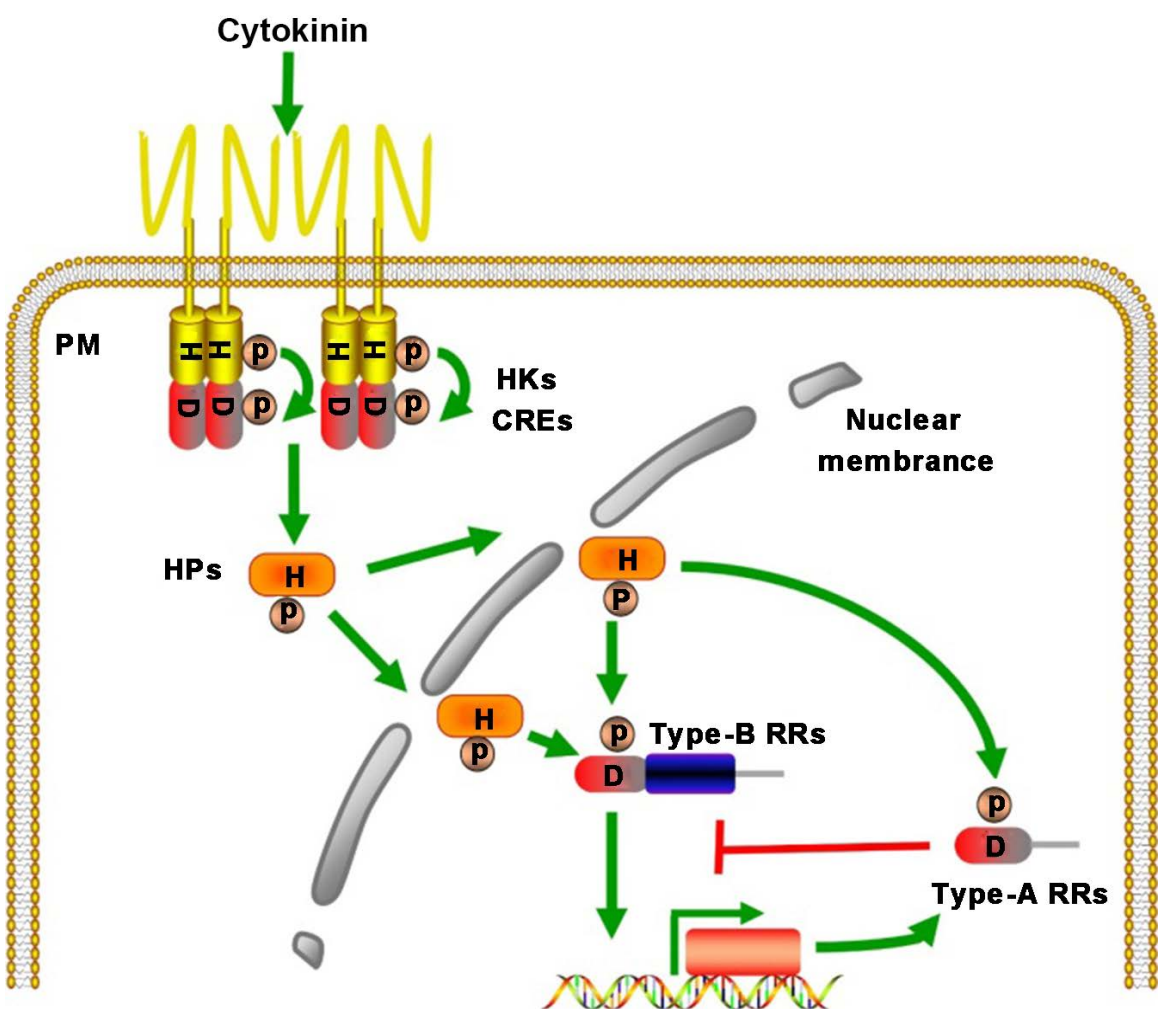

Figure 1. Model of the two-component signal transduction pathway. Cytokinin responses (CREs), histidine-kinases (HKs), histidine phosphotransfer proteins (HPs) and type-B response regulators (RRs) work as a positive regulatory loop and transfer the cytokinin from plasma membrane (PM) to nucleus. Type-B RRs act as transcription factors to regulate some cytokinin targets, including type-A RRs. The type-A RRs can inhibit their own transcription, providing a negative feedback mechanism. H: phospho-accepting histidine residue. D: aspartate residue. P: phosphoric acid groups.

together with position information in melon genome were obtained from $\mathrm{Cu}$ curbit Genomics Database (http://cucurbitgenomics.org/organism/3). The transmembrane domains of melon TCS proteins were analyzed by TMHMM Server v.2.0 (http://www.cbs.dtu.dk/services/TMHMM/).

\subsection{Phylogenetic Analysis and Gene Structure Construction}

Phylogenetic analysis of the full-length protein sequences was conducted using MEGA5 [15]. The evolutionary history was inferred using the Neighbor-Joining method with the following parameters: Poisson correction, pairwise deletion, and bootstrap (1000 replicates) [16] [17]. The DNA and cDNA sequences corresponding to each predicted genes were downloaded from the melon genome database (http://cucurbitgenomics.org/organism/3), and then the gene structures were analyzed using the Gene Structure Display Server online tool (http://gsds.cbi.pku.edu.cn/).

\section{Results and Discussion}

The TCS signaling is widely present in higher plant, including Arabidopsis tha- 
liana [8] [9], rice (Oryza sativa) [18], lotus (Lotus japonicus) [19], soybean (Glycine $\max$ ) [14] [20], maize (Zea maize) [21], Physcomitrella patens [22] [23], and wheat (Triticum aestivum L.) [24], as well as horticultural crops such as Chinese cabbage (Brassica rapa) [20], tomato (Solanum lycopersicum) [25], cucumber (Cucumis sativus L.) [13] and watermelon (Citrullus lanatus) [13] (Table 1). In Arabidopsis, there are 56 TCS genes and their functions have been extensively studied [8] [9]. To find the putative TCS members in melon, we performed a BLASTP search against the melon genome database by using 56 Arabidopsis TCS protein sequences. A total of 90 genes were selected as putative TCS genes including $26 H K(L) s, 6 H P s, 58 R R s$ in the melon genome. To confirm these putative melon TCS genes, the amino acid sequences of all 90 genes were further filtered by Pfam and SMART based on the presence of structural and conserved TCS elements. Finally, 44 typical TCS genes including $18 H K(L) s$, $5 H P S, 21 R R s$ were identified in melon (Table 1). To better reflect the paralogous relationship, all melon TCS members were named according to their homology with Arabidopsis counterparts.

The identified 18 putative $C m H K(L) s$ in melon were separated as $9 \mathrm{CmHKs}$ and $9 \mathrm{CmHKLs}$ according to the presence or absence of conserved residues required for histidine kinase activity (Figure 2(a)). Further they were classified to four distinct gene families: the typical $C m H K$ family (four cytokinin receptor-like $C m H K s$, one $C K I 1$-like $C m H K$, one $C K I 2 / A H K 5$-like $C m H K$, and one $A H K 1$-like $C m H K$ ), the ethylene response (ETR) homolog family (two ETR1-like CmHKs, one ETR2-like CmHKLs), the phytochromes (PHY) (six $P H Y$-like CmHKLs) and the pyruvate dehydrogenase kinase (PDK) family (two $P D K$-like CmHKLs) (Table 2). The protein sequences of these $C m H K(L) s$ ranged from 352 to 1261 amino acids, indicating great variations in their structures and possible functions (Table 2).

Table 1. Summary of the TCS gene number identified in plants.

\begin{tabular}{ccccccccc}
\hline Species & HK(L) & HP (pseudo-HP) & Type-A RR & Type-B RR & Type-C RR & Pseudo RR & Total & References \\
\hline Arabidopsis thaliana & $17(9)$ & $6(1)$ & 10 & 12 & 2 & 9 & 56 & {$[8][9]$} \\
Oryza sativa & $14(8)$ & 5 & 20 & 7 & 0 & 5 & 51 & {$[18]$} \\
Lotus japonicus & 14 & 7 & 7 & 11 & 1 & 5 & 45 & {$[19]$} \\
Glycine max & $36(15)$ & $13(3)$ & 18 & 15 & 3 & 13 & 98 & {$[14][20]$} \\
Zea mays & $11(3)$ & $9(2)$ & 21 & 7 & 0 & 0 & 48 & {$[21]$} \\
Physcomitrella patens & 18 & 3 & 7 & 5 & 4 & 4 & 41 & {$[22][23]$} \\
Triticum aestivum & 7 & 10 & 41 & 2 & 0 & 2 & 62 & {$[24]$} \\
Brassica rapa & $20(9)$ & $8(1)$ & 21 & 17 & 4 & 15 & 85 & {$[20]$} \\
Citrullus lanatus & $19(9)$ & $6(2)$ & 8 & 10 & 1 & 5 & 49 & {$[13]$} \\
Solanum lycopersicum & $20(11)$ & $6(2)$ & 7 & 23 & 1 & 8 & 65 & {$[25]$} \\
Cucumis sativus $\mathrm{L}$ & $18(8)$ & $7(2)$ & 8 & 8 & 0 & 5 & 46 & {$[13]$} \\
Cucumis melon $\mathrm{L}$ & $18(9)$ & $5(1)$ & 7 & 8 & 0 & 6 & 44 & This work \\
\hline
\end{tabular}


(a)
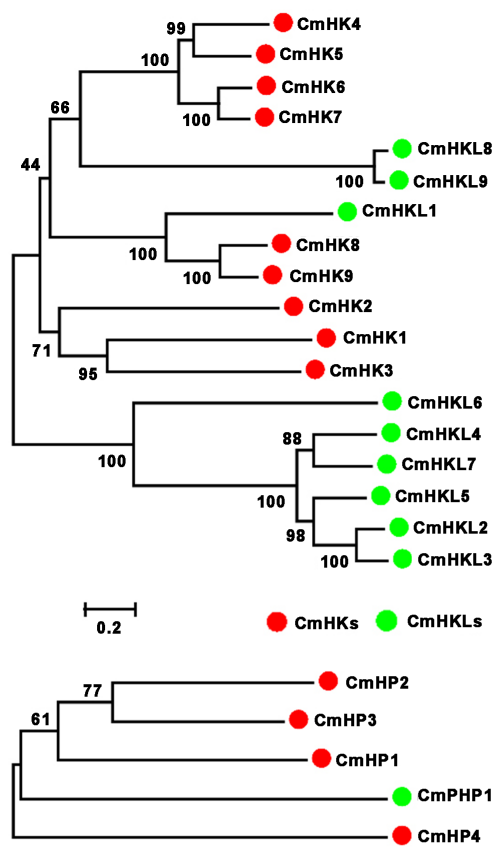

(c)

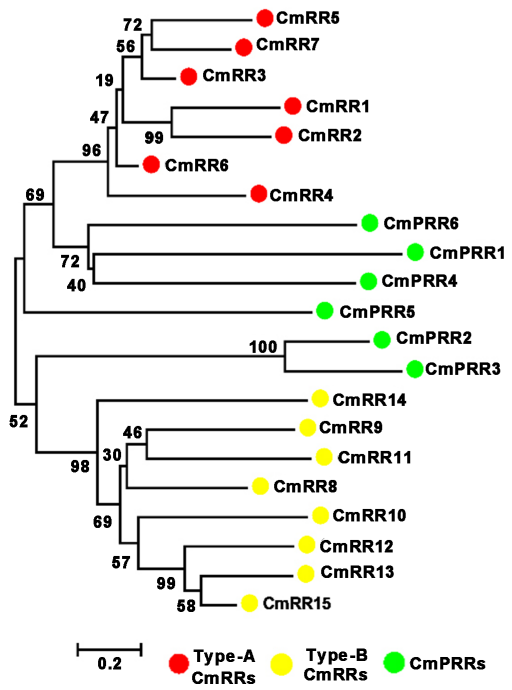

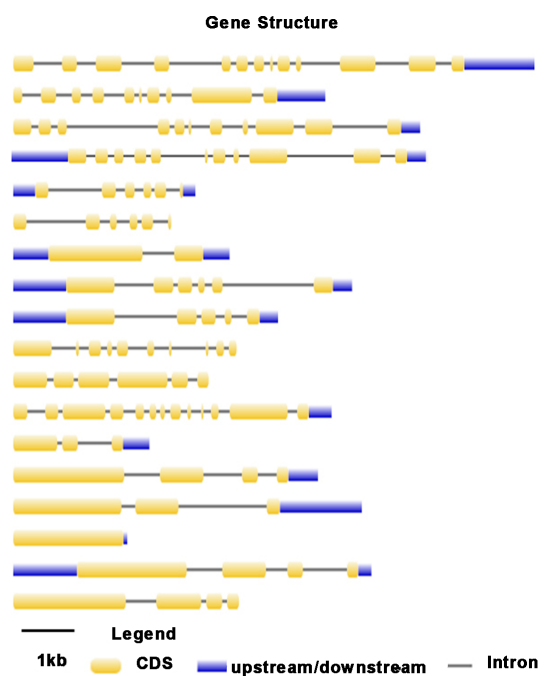

1kb cDs
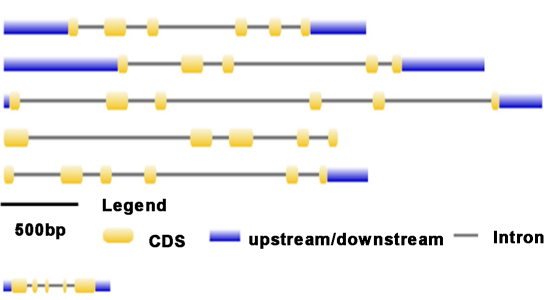

ind

-

$-\cdots=$

a-n-

$-\cdots$

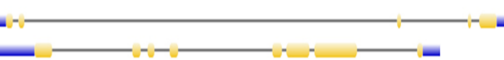

$-1-1-1-1$

- - - - -

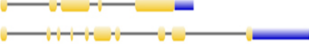

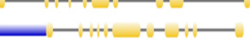

$\cdots-1-$

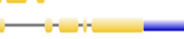

-

- - - - -

$-1-1-$

$-1-1-$

$--$

- Legend

1kb - cDS upstream/downstream - Intron

Figure 2. Phylogenetic relationship and gene structure of the Histidine kinases (a), Histidine phosphotransfer proteins (b) and Response regulators (c) in melon. The phylogenetic tree was constructed based on the Neighbor-Joining method by MEGA5. Bootstrap supports from 1000 replicates were indicated at each branch. The gene structure was analyzed using the Gene Structure Display Server online tool.

Four authentic HPs and one pseudo-HP (PHP) with a pseudo- $H p t$ domain were identified in melon genome (Figure 2(b)). The CmHP1, CmHP2 and CmHP3 had a close relationship with $A H P 1$ (Figure 2(b), Table 3), a positive regulators in CK signaling [26], while the CmHP4 was close to AHP4 (Table 3), which was evolutionarily distinct from the other $A H P s$ and functioned as a negative regulator in CK signaling [26]. CmPHP1 exhibited the longest CDS and 
Table 2. Features of $H K$ genes in melon.

\begin{tabular}{|c|c|c|c|c|c|c|c|c|}
\hline Gene name & Locus $^{\mathrm{a}}$ & Features $^{\mathrm{b}}$ & $\begin{array}{l}\text { NO.of } \\
\mathrm{TM}^{\mathrm{c}}\end{array}$ & Family $^{\mathrm{d}}$ & Chr. & Location & $\begin{array}{l}\text { Length } \\
\text { (CDS) }\end{array}$ & $\begin{array}{c}\text { Length } \\
\text { (AA) }\end{array}$ \\
\hline $\mathrm{CmHK} 1$ & MELO3C004183 & $\mathrm{HK}, \mathrm{Rec}$ & 2 & CKI1 like & 5 & $24012076-24015727$ & 3042 & 1013 \\
\hline CmHK2 & MELO3C016937 & HK & 0 & CKI2/AHK5 like & 7 & 1011644-1015819 & 1827 & 608 \\
\hline $\mathrm{CmHK} 3$ & MELO3C013758 & $\mathrm{HK}, \mathrm{Rec}$ & 2 & AHK1 like & 6 & $35430786-35436735$ & 3678 & 1225 \\
\hline CmHK4 & MELO3C025982 & CHASE, HK, Rec & 3 & AHK2 like & 11 & $13051983-13061724$ & 3786 & 1261 \\
\hline CmHK5 & MELO3C022310 & CHASE, HK, Rec & 1 & AHK3 like & 11 & $29688783-29694615$ & 2799 & 932 \\
\hline CmHK6 & MELO3C020055 & CHASE, HK, Rec & 2 & AHK4 like & 10 & $11370534-11378139$ & 3015 & 1004 \\
\hline CmHK7 & MELO3C005250 & CHASE, HK, Rec & 2 & AHK4 like & 9 & $18109706-18117450$ & 2970 & 989 \\
\hline CmHK8 & MELO3C003906 & GAF, HK, Rec & 3 & ETR1 like & 5 & $19336090-19342422$ & 2223 & 740 \\
\hline CmHK9 & MELO3C015961 & GAF, HK & 3 & ETR1 like & 1 & $30737715-30742663$ & 1914 & 637 \\
\hline CmHKL1 & MELO3C006451 & GAF, HKL, Rec & 4 & ETR2 like & 6 & $3311645-3315687$ & 2304 & 767 \\
\hline CmHKL2 & MELO3C026502 & GAF,PHY,PAS,HKL & 0 & PHYA like & 3 & $22669205-22675899$ & 3372 & 1123 \\
\hline CmHKL3 & MELO3C026506 & GAF,PHY,PAS,HKL & 0 & PHYA like & 3 & $22702766-22706984$ & 3462 & 1153 \\
\hline CmHKL4 & MELO3C006717 & GAF,PHY,PAS,HKL & 0 & PHYB like & 6 & $5314892-5320586$ & 3399 & 1132 \\
\hline CmHKL5 & MELO3C024195 & GAF, PHY, PAS & 0 & PHYC like & 1 & 4097121-4099252 & 2061 & 686 \\
\hline CmHKL6 & MELO3C024196 & PAS, HKL & 0 & PHYC like & 1 & $4094242-4096786$ & 1326 & 441 \\
\hline CmHKL7 & MELO3C002705 & GAF,PHY,PAS,HKL & 0 & PHYE like & 12 & 20683634-20690149 & 3081 & 1026 \\
\hline CmHKL8 & MELO3C006749 & HKL & 0 & PDK like & 6 & $5676982-5680391$ & 1110 & 369 \\
\hline CmHKL9 & MELO3C003205 & HKL & 0 & PDK like & 8 & $30902754-30905707$ & 1059 & 352 \\
\hline
\end{tabular}

Note: a. Systematic names given to genes by Cucurbit Genomics Database. b. Features indicated conserved histidine-kinase (HK) domain, diverged histidine-kinase like (HKL) domain, receiver (Rec) domain, cyclases/histidine kinases associated sensory extracellular (CHASE) domain, cGMP phosphodiesterase/adenylyl cyclase/FhlA (GAF) domain, Per-ARNT-Sim (PAS) domain, and phytochrome (PHY) domain. c. Number of TM (transmembrane) from TMHMM Server v. 2.0 (http://www.cbs.dtu.dk/services/TMHMM/). d. The proteins belonged to which family in Arabidopsis, including cytokinin independent (CKI), Arabidopsis histidine-kinase (AHK), ethylene response (ETR), phytochrome (PHY), and pyruvate dehydrogenase kinase (PDK).

Table 3. Features of $H P$ genes in melon.

\begin{tabular}{|c|c|c|c|c|c|c|c|c|}
\hline Gene name & Locus $^{\mathrm{a}}$ & Features $^{\mathrm{b}}$ & NO.of $\mathrm{TM}^{\mathrm{c}}$ & Family $^{\mathrm{d}}$ & Chr. & Location & $\begin{array}{l}\text { Length } \\
\text { (CDS) }\end{array}$ & Length (AA) \\
\hline CmHP1 & MELO3C015359 & $\mathrm{HPt}$ & 0 & AHP1 like & 2 & 1029369-1032563 & 456 & 151 \\
\hline $\mathrm{CmHP} 2$ & MELO3C006593 & $\mathrm{HPt}$ & 0 & AHP1 like & 6 & 4414993-4417142 & 462 & 153 \\
\hline $\mathrm{CmHP} 3$ & MELO3C021379 & $\mathrm{HPt}$ & 0 & AHP1 like & 11 & $27044881-27047733$ & 393 & 130 \\
\hline $\mathrm{CmHP} 4$ & MELO3C024439 & $\mathrm{HPt}$ & 0 & AHP4 like & 1 & $35201615-35203775$ & 453 & 150 \\
\hline CmPHP1 & MELO3C017877 & Pseudo-HPt & 0 & AHP6 like & 7 & $25647268-25649249$ & 552 & 183 \\
\hline
\end{tabular}

Note: a. Systematic names given to genes by Cucurbit Genomics Database. b. Features included conserved histidine-containing phosphotransfer (HPt) domain and a pseudo-HPt domain lacking the histidine phosphorylation site. c. Number of TM (transmembrane) from TMHMM Server v. 2.0 (http://www.cbs.dtu.dk/services/TMHMM/). d. The proteins belonged to Arabidopsis histidine phosphotransfer (AHP) family.

amino acid sequence (Table 3) and had close relationship with Arabidopsis $A H P 6$, which functioned as a competitor of other $A H P s$ and played a negative role in CK responses by interfering with phosphorelay [27].

There were 21 protein-coding genes in the melon genome that were predicted as $R R s$ (Table 4). Also there were 6 genes encoding $R R \mathrm{~s}$ without the essential residues that were required for biological activity, and were thus named as pseudo-RRs (PRRs) (Table 4). Among these RRs/PRRs, we identified seven type-A $R R s$ (CmRR1-7), each of which contained a Rec domain along with short C-terminal extension (Table 4, Figure 2(c)). Furthermore, these type-A CmRRs exhibited close relationship to their homologs, namely, ARR3, ARR5 and ARR9 in Arabidopsis (Table 4). Genetic analysis suggests that ARR3, ARR5 and ARR9 could function as negative regulators in cytokinin signaling, thus possibly 
Table 4. Features of $R R$ genes in melon.

\begin{tabular}{|c|c|c|c|c|c|c|c|c|}
\hline Gene name & Locus $^{\mathrm{a}}$ & Features $^{\mathrm{b}}$ & $\begin{array}{l}\text { NO.of } \\
\mathrm{TM}^{\mathrm{c}}\end{array}$ & Family $^{\mathrm{d}}$ & Chr. & Location & $\begin{array}{l}\text { Length } \\
\text { (CDS) }\end{array}$ & Length (AA) \\
\hline \multicolumn{9}{|c|}{ Type-A response regulator in Melon } \\
\hline CmRR1 & MELO3C012031 & $\operatorname{Rec}$ & 0 & ARR3 like & 10 & $3154722-3156150$ & 702 & 233 \\
\hline CmRR2 & MELO3C017128 & $\operatorname{Rec}$ & 0 & ARR5 like & 2 & $24899213-24901003$ & 687 & 228 \\
\hline CmRR3 & MELO3C012470 & $\operatorname{Rec}$ & 0 & ARR9 like & 10 & $276889-278626$ & 525 & 174 \\
\hline CmRR4 & MELO3C010624 & $\operatorname{Rec}$ & 0 & ARR9 like & 3 & $8270800-8280939$ & 732 & 243 \\
\hline CmRR5 & MELO3C019056 & $\operatorname{Rec}$ & 0 & ARR9 like & 8 & $10595693-10597773$ & 963 & 320 \\
\hline CmRR6 & MELO3C005156 & $\operatorname{Rec}$ & 0 & ARR9 like & 9 & $16724305-16725377$ & 468 & 155 \\
\hline CmRR7 & MELO3C009770 & $\operatorname{Rec}$ & 0 & ARR9 like & 4 & $28260826-28262276$ & 708 & 235 \\
\hline \multicolumn{9}{|c|}{ Type-B response regulator in Melon } \\
\hline CmRR8 & MELO3C006873 & $\operatorname{Rec}$ & 0 & ARR2 like & 6 & $6879744-6880601$ & 603 & 200 \\
\hline CmRR9 & MELO3C022469 & $\operatorname{Rec}$ & 0 & ARR2 like & 11 & $30889957-30890865$ & 657 & 218 \\
\hline CmRR10 & MELO3C006693 & Rec, Myb & 0 & ARR2 like & 6 & $5116428-5120493$ & 1983 & 660 \\
\hline CmRR11 & MELO3C010714 & Rec, Myb & 0 & ARR11 like & 3 & $29045246-29049016$ & 1755 & 584 \\
\hline CmRR12 & MELO3C016975 & Rec, Myb & 0 & ARR12 like & 7 & $687521-692408$ & 2070 & 689 \\
\hline CmRR13 & MELO3C010245 & Rec, Myb & 0 & ARR12 like & 2 & $15090353-15093797$ & 2028 & 675 \\
\hline CmRR14 & MELO3C017472 & Rec, Myb & 0 & ARR12 like & 2 & $21873514-21875144$ & 1239 & 412 \\
\hline CmRR15 & MELO3C004489 & Rec, Myb & 1 & ARR12 like & 5 & $26950179-26952081$ & 861 & 286 \\
\hline \multicolumn{9}{|c|}{ Pseudo response regulator in Melon } \\
\hline CmPRR1 & MELO3C001999 & Pseudo-Rec, CCT & 0 & APRR1 like & 12 & $25825781-25830612$ & 1671 & 556 \\
\hline CmPRR2 & MELO3C003375 & Pseudo-Rec, Myb & 0 & APRR2 like & 4 & $685095-691112$ & 1371 & 456 \\
\hline CmPRR3 & MELO3C013874 & Pseudo-Rec, Myb & 0 & APRR2 like & 6 & $34410144-34415480$ & 1680 & 559 \\
\hline CmPRR4 & MELO3C005921 & Pseudo-Rec & 0 & APRR5 like & 9 & $24061601-24066514$ & 2091 & 696 \\
\hline CmPRR5 & MELO3C005336 & Pseudo-Rec & 0 & APRR6 like & 9 & 19505431-19509190 & 1686 & 561 \\
\hline CmPRR6 & MELO3C003075 & Pseudo-Rec, CCT & 0 & APRR7 like & 8 & $29585304-29594173$ & 2376 & 791 \\
\hline
\end{tabular}

Note: a. Systematic names given to genes by Cucurbit Genomics Database. b. Features included receiver (Rec) domain, pseudo-receiver (Pseudo-Rec) domain, Myb-like DNA binding domain and CCT (CO, COL and TOC1) motif. c. Number of TM (transmembrane) from TMHMM Server v. 2.0 (http://www.cbs.dtu.dk/services/TMHMM/). d. The proteins belonged to Arabidopsis response regulator (ARR) or Arabidopsis pseudo-response regulator (APRR) family.

participating in a negative feedback loop to reduce the plant sensitivity to cytokinins [28] [25].

There were eight type- $\mathrm{B} R R$ genes in melon, of which 6 members were transcription factors (TFs) and contained long C-terminal extensions with $M Y B$-like DNA binding domains (Table 4). The other two type-B RRs, CmRR 8 and CmRR9, only had Rec domains and their $M Y B$-like domains might be lost during the evolution of the melon $R R$ family (Table 4). These type-B CmRRs shared high sequence similarities to their homologs, $A R R 2, A R R 11$ and $A R R 12$, in Arabidopsis (Table 4). It has been reported that Arabidopsis $A R R 2$ and $A R R 12$ play key roles in ethylene and CK signaling, respectively [29] [30] [31] [32] [33].

Six melon $P R R$ s contained highly-diverged Rec domains (Table 4) and C-terminal extensions. Intriguingly, the CCT-domain and $M Y B$-like domain in type-B RRs were also found in CmPRR1/CmPRR6 and CmPRR2/CmPRR3, respectively. However, the exception occurred to the CmPRR4 and CmPRR5, which lacked both the CCT and the $M Y B$-like domains (Table 4). These great divergences should be paid more attentions on in the future study. 


\section{Acknowledgements}

This work was supported by National Natural Science Foundation of China (31401894 and 31501781), “Taishan Scholar" Foundation of the People's Government of Shandong Province, and China Postdoctoral Science Foundation (2017M612741).

\section{References}

[1] Hwang, I, Sheen, J., and Müller, B. (2012) Cytokinin Signaling Networks. Annual Review of Plant Biology, 63, 353-380. https://doi.org/10.1146/annurev-arplant-042811-105503

[2] Sasaki, T., Suzaki, T., Soyano, T., Kojima, M., Sakakibara, H., and Kawaguchi, M. (2014) Shoot-Derived Cytokinins Systemically Regulate Root Nodulation. Nature Communications, 5, 4983. https://doi.org/10.1038/ncomms5983

[3] Nitschke, S., Cortleven, A., Iven, T., Feussner, I., Havaux, M., Riefler, M. and Schmülling, T. (2016) Circadian Stress Regimes Affect the Circadian Clock and Cause Jasmonic Acid-Dependent Cell Death in Cytokinin-Deficient Arabidopsis Plants. Plant Cell, 28, 1616-1639. https://doi.org/10.1105/tpc.16.00016

[4] Kang, J., Lee, Y., Sakakibara, H. and Martinoia, E. (2017) Cytokinin Transporters: Go and Stop in Signaling. Trends in Plant Science, 22, 455-461. https://doi.org/10.1016/j.tplants.2017.03.003

[5] Thomason, P. and Kay, R. (2000) Eukaryotic Signal Transduction via Histidine-Aspartate Phosphorelay. Journal of Cell Science, 113, 3141-3150.

http://jcs.biologists.org/content/joces/113/18/3141.full.pdf

[6] Urao, T., Yamaguchi-Shinozaki, K., and Shinozaki, K. (2000) Two-Component Systems in Plant Signal Transduction. Trends in Plant Science, 5, 67-74. https://doi.org/10.1016/S1360-1385(99)01542-3

[7] Stock, A.M., Robinson, V.L. and Goudreau, P.N. (2000) Two-Component Signal Transduction. Annual Review of Biochemistry, 69, 183-215. https://doi.org/10.1146/annurev.biochem.69.1.183

[8] Hwang, I., Chen, H.C. and Sheen, J. (2002) Two-Component Signal Transduction Pathways in Arabidopsis. Plant Physiology, 129, 500-515. https://doi.org/10.1104/pp.005504

[9] Schaller, G.E., Kieber, J.J. and Shiuc, S.H. (2008) Two-Component Signaling Elements and Histidyl-Aspartyl Phosphorelays. Arabidopsis Book, 6, e0112. https://doi.org/10.1199/tab.0112

[10] Garcia-Mas, J., Benjak, A., Sanseverino, W., Bourgeois, M., Mir, G., González, V.M., Hénaff, E., Câmara, F., Cozzuto, L., Lowy, E., Alioto, T., Capella-Gutiérrez, S., Blanca, J., Cañizares, J., Ziarsolo, P., Gonzalez-Ibeas, D., Rodríguez-Moreno, L., Droege, M., Du, L., Alvarez-Tejado, M., Lorente-Galdos, B., Melé, M., Yang, M. Weng, Y.Q., Navarro, A., Marques-Bonet, T., Aranda, M.A., Nuez, F., Picó, B., Gabaldón, T., Roma, G., Guigó, R., Casacuberta, J.M., Arús, P. and Puigdomènech., P. (2012) The Genome of Melon (Cucumis melo L.). PNAS, 109, 11872-11877. https://doi.org/10.1073/pnas.1205415109

[11] Díaz, A., Martín-Hernández, A.M., Dolcett-Sanjuan, R., Garcés-Claver, A., Álvarez, J.M., Garcia-Mas, J., Picó, B. and Monforte, A.J. (2017) Quantitative Trait Loci Analysis of Melon (Cucumis melo L.) Domestication-Related Traits. Theoretical and Applied Genetics, 130, 1837-1856. https://doi.org/10.1007/s00122-017-2928-y 
[12] Chang, C.W., Wang, Y.H. and Tung, C.W. (2017) Genome-Wide Single Nucleotide Polymorphism Discovery and the Construction of a High-Density Genetic Map for Melon (Cucumis melo L.) Using Genotyping-by-Sequencing. Frontiers in Plant Science, 8, 125. https://doi.org/10.3389/fpls.2017.00125

[13] He, Y.J., Liu, X., Zou, T., Pan, C.T., Qin, L., Chen, L.F. and Lu, G. (2016) Genome-Wide Identification of Two-Component System Genes in Cucurbitaceae Crops and Expression Profiling Analyses in Cucumber. Frontiers in Plant Science, 7, 899. https://doi.org/10.3389/fpls.2016.00899

[14] Mochida, K., Yoshida, T., Sakurai, T., Yamaguchi-Shinozaki, K., Shinozaki, K. and Tran, L.P. (2010) Genome-Wide Analysis of Two-Component Systems and Prediction of Stress-Responsive Two-Component System Members in Soybean. DNA Research, 17, 303-324. https://doi.org/10.1093/dnares/dsq021

[15] Tamura, K., Peterson, D., Peterson, N., Stecher, G., Nei, M. and Kumar, S. (2011) MEGA5: Molecular Evolutionary Genetics Analysis Using Maximum Likelihood, Evolutionary Distance, and Maximum Parsimony Methods. Molecular Biology and Evolution, 28, 2731-2739. https://doi.org/10.1093/molbev/msr121

[16] Saitou, N. and Nei, M. (1987) The Neighbor-Joining Method: A New Method for Reconstructing Phylogenetic Trees. Molecular Biology and Evolution, 4, 406-425.

[17] Li, Q., Zhao, P., Li, J., Zhang, C., Wang, L. and Ren Z. (2014) Genome-Wide Analysis of the WD-Repeat Protein Family in Cucumber and Arabidopsis. Molecular Genetics and Genomics, 289, 103-124. https://doi.org/10.1007/s00438-013-0789-x

[18] Pareek, A., Singh, A., Kumar, M., Kushwaha, H.R., Lynn, A.M. and Singla-Pareek, S.L. (2006) Whole-Genome Analysis of Oryza sativa Reveals Similar Architecture of Two-Component Signaling Machinery with Arabidopsis. Plant Physiology, 142, 380-397. https://doi.org/10.1104/pp.106.086371

[19] Ishida, K., Niwa, Y., Yamashino, T. and Mizuno, T. (2009) A Genome-Wide Compilation of the Two-Component Systems in Lotus japonicus. DNA Research, 16, 237-247. https://doi.org/10.1093/dnares/dsp012

[20] Liu, Z., Zhang, M., Kong, L., Lv, Y., Zou, M., Lu, G., Cao, J.S. and Yu, X.L. (2014) Genome Wide Identification, Phylogeny, Duplication, and Expression Analyses of Two Component System Genes in Chinese Cabbage (Brassica rapa ssp. pekinensis). DNA Research, 21, 379-396. https://doi.org/10.1093/dnares/dsu004

[21] Chu, Z.X., Ma, Q., Lin, Y.X., Tang, X.L., Zhou, Y.Q., Zhu, S.W., Fan, J. and Cheng, B.J. (2011) Genome-Wide Identification, Classification, and Analysis of Two-Component Signal System Genes in Maize. Genetics and Molecular Research, 10, 3316-3330. https://doi.org/10.4238/2011.December.8.3

[22] Ishida, K., Yamashino, T., Nakanishi, H. and Mizuno, T. (2010) Classification of the Genes Involved in the Two-Component System of the Moss Physcomitrella patens. Bioscience, Biotechnology, and Biochemistry, 74, 2542-2545. https://doi.org/10.1271/bbb.100623

[23] Satbhai, S.B., Yamashino, T., Okada, R., Nomoto, Y., Mizuno, T., Tezuka, Y., Itoh, T., Tomita, M., Otsuki, S. and Aoki, S. (2011) Pseudo-Response Regulator (PRR) Homologues of the Moss Physcomitrella Patens: Insights into the Evolution of the PRR Family in Land Plants. DNA Research, 18, 39-52. https://doi.org/10.1093/dnares/dsq033

[24] Gahlaut, V., Mathur, S., Dhariwal, R., Khurana, J.P., Tyagi, A.K., Balyan, H.S. and Gupta, P.K. (2014) A Multi-Step Phosphorelay Two-Component System Impacts on Tolerance against Dehydration Stress in Common Wheat. Functional \& Integrative Genomics, 14, 707-716. https://doi.org/10.1007/s10142-014-0398-8 
[25] He, Y.J., Liu, X., Ye, L., Pan, C.T., Chen, L.F., Zou, T. and Lu, G. (2016) Genome-Wide Identification and Expression Analysis of Two-Component System Genes in Tomato. International Journal of Molecular Sciences, 17, 1204. https://doi.org/10.3390/ijms17081204

[26] Hutchison, C.E., Li, J., Argueso, C., Gonzalez, M., Lee, E., Lewis, M.W., Maxwell, B.B., Perdue, T.D., Schaller, G.E., Alonso, J.M., Ecker, J.R. and Kieber, J.J. (2006) The Arabidopsis Histidine Phosphotransfer Proteins Are Redundant Positive Regulators of Cytokinin Signaling. Plant Cell, 18, 3073-3087. https://doi.org/10.1105/tpc.106.045674

[27] Mähönen, A.P., Bishopp, A., Higuchi, M., Nieminen, K.M., Kinoshita, K., Törmäkangas, K., Ikeda, Y., Oka, A., Kakimoto, T. and Helariutta, Y. (2006) Cytokinin Signaling and Its Inhibitor AHP6 Regulate Cell Fate during Vascular Development. Science, 311, 94-98. https://doi.org/10.1126/science.1118875

[28] To, J.P., Haberer, G., Ferreira, F.J., Deruere, J., Mason, M.G., Schaller, G.E., Alonso, J.M., Ecker, J.R. and Kieber, J.J. (2004) Type-A Arabidopsis Response Regulators Are Partially Redundant Negative Regulators of Cytokinin Signaling. Plant Cell, 16, 658-671. https://doi.org/10.1105/tpc.018978

[29] To, J.P.C., Deruere, J., Maxwell, B.B., Morris, V.F., Hutchison, C.E., Ferreira, F.J., Schaller, G.E. and Kieber, J.J. (2007) Cytokinin Regulates Type-A Arabidopsis Response Regulator Activity and Protein Stability via Two-Component Phosphorelay. Plant Cell, 19, 3901-3914. https://doi.org/10.1105/tpc.107.052662

[30] Mason, M.G., Li, J., Mathews, D.E., Kieber, J.J. and Schaller, G.E. (2004) Type-B Response Regulators Display Overlapping Expression Patterns in Arabidopsis. Plant Physiology, 135, 927-937. https://doi.org/10.1104/pp.103.038109

[31] Yokoyama, A., Yamashino, T., Amano, Y., Tajima, Y., Imamura, A., Sakakibara, H. and Mizuno, T. (2007) Type-B ARR Transcription Factors, $A R R 10$ and $A R R 12$, Are Implicated in Cytokinin-Mediated Regulation of Protoxylem Differentiation in Roots of Arabidopsis thaliana. Plant and Cell Physiology, 48, 84-96. https://doi.org/10.1093/pcp/pcl040

[32] Ishida, K., Yamashino, T., Yokoyama, A. and Mizuno, T. (2008) Three Type-B Response Regulators, $A R R 1, A R R 10$, and $A R R 12$, Play Essential But Redundant Roles in Cytokinin Signal Transduction throughout the Life Cycle of Arabidopsis thaliana. Plant and Cell Physiology, 49, 47-57. https://doi.org/10.1093/pcp/pcm 165

[33] Hass, C., Lohrmann, J., Albrecht, V., Sweere, U., Hummel, F., Yoo, S.D., Hwang, I., Zhu, T., Schafer, E., Kudla, J. and Harter, K. (2004) The Response Regulator $2 \mathrm{Me}-$ diates Ethylene Signalling and Hormone Signal Integration in Arabidopsis. The EMBO Journal, 23, 3290-3302. https://doi.org/10.1038/sj.emboj.7600337 


\section{Appendix}

Table A1. Information of abbreviation notation.

\begin{tabular}{cc}
\hline Abbreviation & Annotation \\
\hline CCT & CO, COL and TOC1 \\
CHASE & cyclases/histidine kinases associated sensing extracellular \\
CK & cytokinin \\
CKI & cytokinin independent \\
CRE & cytokinin response \\
ETR & ethylene response \\
GAF & cGMP phosphodiesterase/adenylyl cyclase/FhlA \\
HATPase & histidine kinase-like ATPase \\
HisKA & His Kinase A (phosphoacceptor) domain \\
HK & histidine kinase \\
HKL & histidine-kinase like \\
HP & histidine phosphotransfer \\
HPt & response regulator \\
PAS & two-component system \\
PDK & transmembrane \\
PHY & pyruvate dehydrogenase kinase \\
PM & phytochrome \\
Rec & plasmembrane \\
RR & TCS
\end{tabular}

